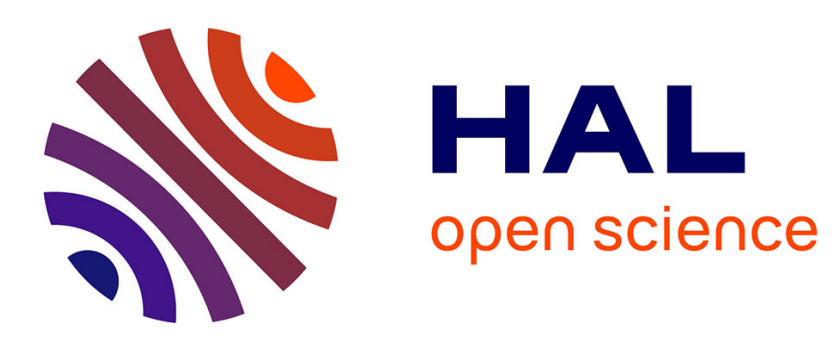

\title{
L'expertise judiciaire dans la construction du jugement : de la ressource à la contrainte
}

Laurence Dumoulin

\section{To cite this version:}

Laurence Dumoulin. L'expertise judiciaire dans la construction du jugement: de la ressource à la contrainte. Droit et Société, 2000, 44-45, pp.199-223. halshs-00153331

\section{HAL Id: halshs-00153331 \\ https://shs.hal.science/halshs-00153331}

Submitted on 20 Nov 2009

HAL is a multi-disciplinary open access archive for the deposit and dissemination of scientific research documents, whether they are published or not. The documents may come from teaching and research institutions in France or abroad, or from public or private research centers.
L'archive ouverte pluridisciplinaire HAL, est destinée au dépôt et à la diffusion de documents scientifiques de niveau recherche, publiés ou non, émanant des établissements d'enseignement et de recherche français ou étrangers, des laboratoires publics ou privés. 
Article paru dans Droit et société, 44-45, 2000, p.199-223.

L'expertise judiciaire dans la construction du jugement :

de la ressource à la contrainte

\author{
Laurence Dumoulin*
}

* Centre de recherche sur le politique, l'administration, la ville et le territoire, CERAT-IEP, BP 48, 38040 Grenoble cedex 9 . 
«Les ordalies, puis la torture, puis le jury, bientôt l'expertise : tels ont été ou seront les talismans successifs imaginés pour la découverte du vrai en justice » ${ }^{1}$ affirmait à la fin du siècle dernier Gabriel Tarde. Favorable à l'avènement d'une Justice plus scientifique, il considérait en effet qu' « un siècle qui se dit savant se doit à lui-même [...] de juger savamment ses délits »². Engagé à l'époque où Gabriel Tarde écrit ces lignes, le processus d’ouverture de la Justice aux experts s'est poursuivi tout au long du XXe siècle ${ }^{3}$. L’institution judiciaire n’a cessé de s’entourer, pour rendre la justice, de techniciens aux spécialités de plus en plus diverses : médecine légale, arpentage, horlogerie, comptabilité, architecture, informatique, etc.

Ces partenaires d’un genre particulier se sont installés dans l’espace judiciaire, pénétrant au cœur même de ce qui constitue l'essence et la quintessence de l’activité de justice : le jugement. Or, bien que banalisée par le discours juridique, cette présence de savoirs et savoir-faire extérieurs au sein de la Justice, interroge fortement les fondements de cette institution qui, au nom du droit et de la légalité, détient le pouvoir de juger. Dès lors que des logiques profanes sont introduites au plus profond du judiciaire, qu'en est-il du monopole du droit dans la définition du juste ${ }^{4}$ ?

Nécessaire dans le cadre d'une institution soucieuse de rationaliser ses modes d'action et ses décisions, appelée de ses vœux par une «société critique » ${ }^{5}$ en émergence, cette ouverture aux sciences et techniques place désormais le droit, la raison juridique, en concurrence avec d'autres modes de formation et de justification de la parole judiciaire. Que produit alors la confrontation d'un savoir "déjà-là", transcendant et à vocation totalisante avec des savoirs exogènes, profanes mais néanmoins nécessaires ? Quelles places respectives occupent les disciplines non juridiques et

\footnotetext{
${ }^{1}$ Gabriel TARDE, La philosophie pénale, Paris, éd. Cujas, coll. « Bibliothèque internationale de criminologie », 1972 ( $4^{\mathrm{e}}$ éd.), p. 436. (La première parution date de 1890.)

${ }^{2}$ Ibid. p. 453.

${ }^{3}$ Frédéric CHAUVAUD av. la collab. de Laurence DUMOULIN, Experts et expertises judiciaires en France (17911944), Rapport Gerhico / GIP Justice, Poitiers, ronéotypé, octobre 1999.

${ }^{4}$ On notera que la question vaut également pour le jury de Cour d'assises. Voir Louis GRUEL, Pardons et châtiments. Les jurés français face aux violences criminelles, Paris, Nathan, coll. « Essais \& Recherches », 1991.

${ }^{5}$ Luc BOLTANSKI, « Sociologie critique et critique de la sociologie », Politics, n¹0, 1990, p. 130.
} 
le droit dans la construction du jugement ? De quel partage des tâches entre le magistrat et l'expert naît la décision de justice ?

L'expertise, en tant que forme privilégiée de développement des savoirs dans l'institution judiciaire, offre un angle intéressant et inédit pour reconsidérer à la fois le rapport du droit aux autres disciplines et celui du magistrat au jugement ${ }^{6}$. Alors que la loi conçoit l'expertise comme un accessoire, c'est bien la capacité à produire des certitudes qui, in fine, détermine le niveau de participation du technicien dans l'élaboration du jugement. Tantôt négligeable, tantôt décisive, l'expertise contribue très inégalement à la formation du verdict mais fait varier d'autant le rôle du magistrat. Maître de la décision, le juge peut aussi n'en être que l'organisateur. L'ouverture de la Justice à des partenaires extérieurs donne ainsi à penser une possible évolution des modes de juger pratiqués au sein de cette institution.

L'expertise judiciaire, entre approche praticienne et discours juridique

L'expertise judiciaire constitue, à ce jour, un angle mort des sciences sociales ${ }^{7}$, en particulier de la science politique. Si des approches historiques ${ }^{8}$, sociologiques ${ }^{9}$, psychologiques $^{10}$ ou linguistiques ${ }^{11}$ se développent timidement depuis une quinzaine d'années, la science politique

\footnotetext{
${ }^{6}$ Cet article s’inscrit dans une recherche plus large qui porte sur la dynamique savoir-pouvoir observée à travers le cas de l'expertise judiciaire. Il s'agit d'une thèse de doctorat en science politique, intitulée « L'expertise comme nouvelle raison politique ? Un terrain privilégié : l'expertise judiciaire » et réalisée sous la direction de Jacques Commaille et Martine Kaluszynski. L’auteur tient à remercier chaleureusement les personnes qui lui ont fait l'amitié de relire les différentes versions de ce texte et de lui faire part de leurs remarques aussi judicieuses que bienveillantes : Jacques Commaille, Martine Kaluszynski, Kristoff Talin et Philippe Warin. L'auteur tient également à remercier toutes les personnes qui ont rendu possible le travail de terrain : experts, magistrats et juristes rencontrés.

${ }^{7}$ Il s'agit ici de l'expertise judiciaire en tant qu'objet à part entière. En revanche, les recherches concernant des pans particuliers de l'expertise ne sont pas rares. C'est le cas en particulier de l'expertise médico-légale et psychiatrique. Voir les travaux de Michel FOUCAULT et notamment Les anormaux. Cours au Collège de France. 1974-1975, Paris, Gallimard / Seuil, 1999. Voir également Robert CASTEL, L'ordre psychiatrique, Paris, Ed. de Minuit, 1977 ainsi que Martine KALUSZYNSKI, «Identités professionnelles, identités politiques: médecins et juristes face au crime au tournant du XIXe et du XXe siècle », in Laurent MUCHIELLI (dir.), Histoire de la criminologie française, Paris, L'Harmattan, 1994, p.

${ }^{8}$ Frédéric CHAUVAUD av. la collab. de Laurence DUMOULIN, op. cit.

${ }^{9}$ Philippe FRITSCH, « Situations d'expertise et expert système », in CRESAL, Situations d'expertise et socialisation des savoirs, Saint-Etienne, ronéotypé, 1985, p. 15-47.

${ }^{10}$ Loïck M. VILLERBU et Jean-Luc VIAUX (dir.), Ethique et pratiques psychologiques de l'expertise, Paris, L’Harmattan, coll. « Psychologiques », 1998.

11 Monique de BONIS, L'analyse du rôle de l'expertise dans le processus décisionnel judiciaire observé essentiellement du point de vue cognitif, Rapport pour le Ministère de la Justice, ronéotypé, ss date ; « Langage naturel
} 
française, dans son ensemble, ignore encore très largement cet objet. Le thème général de l'expertise suscite certes de nombreux travaux qui s'appuient sur des terrains de recherche diversifiés. Cependant, le champ judiciaire reste manifestement à l'écart de cet engouement. L'expertise judiciaire ne donne guère lieu à des recherches empiriques, de même qu'elle n'est qu'effleurée par la réflexion théorique.

On dispose en revanche, sur cette question, d’une abondante littérature issue, soit des experts eux-mêmes, soit des juristes. Les manuels d'experts, pour leur part, envisagent surtout l'expertise comme un ensemble de procédés, de manières de faire singulières. L'expertise est alors vue comme une pratique qui articule un savoir disciplinaire donné et une question concrète, ce, dans le respect d'un cadre juridique et procédural vu comme contraignant. La notion de savoir-faire est considérée comme fondatrice de la compétence expertale. Elle repose également sur le respect des exigences juridiques et judiciaires. Les approches praticiennes valorisent ainsi l'activité expertale comme réponse à une commande. En ce sens, elles désamorcent, à la racine, les questionnements et bouleversements que l'appel aux disciplines savantes serait susceptible d’introduire dans la machine judiciaire.

De même, le discours juridique livre une approche "neutralisante" de l'expertise. Pour le droit, l'expertise correspond à une procédure étroite, organisée par le Code de procédure pénale et le Nouveau code de procédure civile d'une part, par la loi relative aux experts judiciaires et son décret d'application ${ }^{12}$ d'autre part. Les contours de cette mesure d'instruction sont méticuleusement tracés par ces différents textes : l'expertise «n’a lieu d'être ordonnée que dans le cas où des constatations ou une consultation ne pourraient suffire à éclairer le juge ${ }^{13}$.

\footnotetext{
et expertise psychiatrique. Les marques de quantité dans la description des sujets expertisés : précision ou exactitude », Droit et société, n³, 1986, p. 251-260 ainsi que Monique de BONIS et Danièle BOURCIER, Les paradoxes de l'expertise. Savoir ou juger ?, Le Plessis-Robinson, Institut Synthélabo pour le progrès de la connaissance, coll. « Les empêcheurs de penser en rond », 1999.

${ }^{12}$ Il s’agit de la loi n71-498 du 29 juin 1971 et du décret n74-1181 du 31 décembre 1974.

${ }^{13}$ Article 263 du Nouveau code de procédure civile.
} 
Mais l'approche juridique n'est pas seulement limitative, elle est aussi volontairement "enfermante". Mesurant les éventuelles perturbations entraînées par une utilisation des savoirs dans le jugement, le "législateur" a réduit l'expertise à une simple forme procédurale, dénuée de tout impact sur la formation du verdict. A l'instar des approches praticiennes, l'approche juridique passe donc sous silence les enjeux que posent l’introduction de disciplines extérieures, au sein de la Justice. Le discours du droit, lui-même porteur d'enjeux, restreint à dessein l'importance et les effets possibles d'une présence des savoirs dans le processus judiciaire. De ce point de vue, les orientations données aux dispositifs formels sont sans équivoque. En effet, les textes normatifs encadrent étroitement les conditions de réalisation de l'expertise, placent l'expert dans un rapport de subordination avec le magistrat et promeuvent l'instrumentalisation de la parole expertale.

Par ailleurs, si le droit a le pouvoir d'énoncer ce qui doit être, il ne peut pour autant contraindre complètement le réel. L'approche juridique, qui raisonne en termes de règle et de conformité à la règle, ne peut rendre compte de cette portion de réalité qui s'échappe précisément des "impératifs" juridiques. Les pratiques juridictionnelles, certes inspirées par la conception juridique de l'expertise, n'en sont pas pour autant calquées sur elle. Elles donnent au contraire l'image d'une réalité nuancée, jouant avec la règle. C'est bien ce regard centré non sur la loi mais sur les jeux autour de la loi, que nous entendons porter ici.

L'expertise judiciaire, l'intérêt d'une approche en termes de pouvoir

Pour construire en objet de recherche cette question particulièrement saturée par le discours praticien, il convient tout d’abord d'en proposer une définition singulière et élargie.

L'expertise judiciaire peut être entendue, dans un sens générique et extensif, comme l'ensemble des formes que prend l'introduction d'une rationalité technico-scientifique dans l'institution, le processus et la décision judiciaires. Contrairement aux autres catégories du personnel en place dans les juridictions, l'expert n'est pas un professionnel du droit. Son identité se construit autour de la maîtrise d’une spécialité technique ou scientifique. Cependant, ce technicien 
prend part au déroulement de l'instance et à la préparation du jugement, que ce soit en matière d’administration de la preuve, d'établissement des faits ou d'évaluation des responsabilités. Aussi peut-on considérer les experts judiciaires comme des acteurs technico-scientifiques qui, en dépit d’une légitimité non juridique, interviennent dans les mécanismes judiciaires et plus spécialement dans la construction du jugement.

L'activité expertale, selon cette acception, est ici travaillée à travers les différentes formes et manifestations qu'elle peut prendre. Elle englobe alors l'ensemble des investigations à caractère technique ou scientifique effectivement intervenues dans le processus judiciaire, que ce soit dans le cadre de l'enquête préliminaire, de l'instruction ou du jugement ; qu’elles soient effectuées d'office, à la demande du Parquet, de la juridiction d'instruction ou de jugement, ou encore des parties ; qu'elles prennent la forme d'observations, d'investigations, de diagnostic ou de conseil. En effet, ce qui importe ce n’est pas tant le cadre juridique, le moment procédural ou l'identité du commissaire que les recherches entreprises, en tant qu'elles viennent effectivement nourrir le dossier judiciaire et la réflexion du magistrat.

Relativisant le discours du droit, nous montrerons que le fonctionnement quotidien de la Justice use et ruse avec souplesse des "impératifs" juridiques. La volonté exprimée par le droit de restreindre le périmètre d'action des savoirs et de veiller à leur innocuité sur les principes et modes d'action de l'institution, témoigne précisément du caractère sensible, névralgique de l'expertise. C'est bien parce qu'il y a "péril en la demeure" que les savoirs sont enfermés dans un véritable carcan procédural. Le danger est d'ailleurs annoncé par certains juristes et praticiens du droit qui évoquent le pouvoir croissant des experts et leur influence grandissante sur l'issue des procès ${ }^{14}$.

Aussi convient-il de restaurer la dimension qu'évitent précisément les dispositifs formels et les commentaires de doctrine et qui trahit leur inconfort : de quels enjeux la présence des savoirs dans le jugement est-elle porteur ? Incontestablement, ce sont bien les pouvoirs respectifs du

\footnotetext{
${ }^{14}$ Voir notamment Jean PIERRE, « Le métier de juge d'instruction », Etudes, 1988, p. 43-52.
} 
magistrat et de l'expert dont il est ici question, avec pour toile de fond le risque d'un gouvernement des experts et / ou de ce qui peut être qualifié comme une démission des juges.

Restaurant pleinement les enjeux propres à ce que le droit présente comme une simple procédure, on s'interroge ici sur les effets, les répercussions de l'expertise dans et sur l'institution judiciaire $^{15}$. Quel rôle le discours expertal joue-t-il dans la décision de justice ? Comment est-il perçu et exploité par les acteurs du mécanisme décisionnel (magistrats, avocats, parties) ? Est-il délaissé, contourné, instrumentalisé ou encore vécu comme une imposition ? La question des usages de la parole expertale doit en effet être posée dans la mesure où elle ouvre directement sur la question des pouvoirs respectifs du magistrat et de l'expert dans la formulation et la justification du jugement. Le rôle des savoirs sera ainsi envisagé sous l'angle des rapports de pouvoir qu'entretiennent hommes de l'art, magistrats et avocats et, à travers eux, disciplines savantes et droit.

Si le droit crée un lien statutaire de subordination entre juge et technicien qui cadre d'emblée la relation, c'est bien au fil des interactions concrètes que se définit la position de chacun. Les relations de pouvoir dynamiques que tissent magistrat et expert, en particulier, s'agencent et s’organisent différemment suivant les contextes. Elles produisent des situations hétérogènes depuis le cas où l'expertise est complètement délaissée et ignorée dans le processus décisionnel jusqu’au cas où la décision découle entièrement des résultats expertaux.

\section{Au fil de la démonstration}

Le droit place l'expertise dans un cadre normatif étroit voire étriqué. Il minimise - pour mieux la limiter - l'influence des savoirs experts dans l'élaboration du jugement. Certaines

\footnotetext{
${ }^{15}$ Notre matériau est constitué de textes juridiques, d'entretiens, d’archives ainsi que d'études de cas à partir de dossiers jugés dans lesquels une ou plusieurs expertises ont été ordonnées et réalisées. Dans le cadre du présent article, notre analyse porte sur des dossiers issus de juridictions civiles de droit commun (le Tribunal de grande instance et la Cour d'appel de Grenoble) ainsi que d'une juridiction d'exception, choisie tant pour son lien particulier à l'expertise que pour des raisons d'accès aux sources (le juge des loyers commerciaux, TGI de Grenoble). Un système de classement et de codage des dossiers a été élaboré afin de citer certaines pièces sans compromettre l'anonymat des parties en présence. Précisons enfin que cet article donne à voir quelques résultats formulés à partir d’un premier dépouillement
} 
pratiques juridictionnelles observées s'inscrivent dans ce modèle de l'expertise ressource exploitée. Les acteurs du processus judiciaire instrumentalisent l'expertise, à l'appui de leurs stratégies discursives (I). Pour autant, on ne peut se contenter de cette vision partielle. La mise en œuvre des dispositifs formels s'effectue également selon d'autres modalités, non prévues par le droit. Apparaît alors le modèle d'une expertise contraignante, s'imposant au juge et le privant d'une partie de sa marge de manœuvre (II).

\section{L'expertise judiciaire : une ressource librement exploitée}

Si certains juristes considèrent l'expertise comme une collaboration entre le juge et l'expert, rejoignant ainsi la position et la pratique de certains magistrats, les textes normatifs instaurent plutôt une relation d'inégalité, de commanditaire à commandité, de maître d'ouvrage à maître d'œuvre. Cette conception de l'expertise issue des dispositifs formels oriente, imprime certaines pratiques observables dans les juridictions. Les acteurs judiciaires puisent dans le rapport d'expertise de quoi étayer leurs positions et argumentations. La "pêche" qu'ils effectuent peut devenir "miraculeuse" lorsque la parole expertale devient le support unique du jugement.

\subsection{L'expertise, une relation d'inégalitée ${ }^{16}$}

Les rapports entre magistrats et experts constituent un des nœuds du discours juridique relatif à l'expertise. Le technicien et le juge sont envisagés dans un étroit lien d’interdépendance. Comme le soulignent les articles de doctrine, «juge et expert forment [...] un couple indissociable ${ }^{17}$. «Tout au long de l'exécution de la mission, ils doivent former un véritable binôme [...] pour s'entraider, s'épauler, avoir recours l'un à l'autre. " ${ }^{18}$ "Entre le juge qui donne la mission et l'expert qui fournit son rapport, c'est un authentique pacte de confiance qui

des matériaux relatifs au civil. Ces premiers acquis demanderont certainement à être nuancés, affinés et comparés avec les observations tirées de la matière pénale.

${ }^{16}$ Comme le dit un éminent juriste, "l'expert collabore avec le juge, sous son autorité »! Marcel CARATINI, op. cit. p. 44.

${ }^{17}$ Marcel CARATINI, « Experts et expertise dans la législation civile française. Principes généraux », La gazette du palais, Doctrine, 22 janvier 1985, p. 44. 
s'instaure. ${ }^{19}$ Cependant, le partenariat et la coopération n’impliquent pas l’égalité des parties en présence. Collaborer, c’est travailler ensemble dans un but partagé mais sans que cela ne préjuge du rapport de force susceptible de s’instaurer entre les partenaires.

Les dispositifs formels qui encadrent la procédure expertale dessinent une architecture verticale, dans laquelle le magistrat contrôle le technicien. «[L]es rôles sont clairement définis : le juge pose une question d'ordre technique à l'expert, l'expert examine les faits et apporte une réponse, le juge tranche en toute liberté ${ }^{20}$. De cette conception décisionniste est issue une relation construite autour d'une inégalité statutaire et d'un rapport de domination. L’un impulse, commandite, ordonne, tandis que l'autre réagit, répond, obéit. La répartition des tâches est division du travail mais aussi et peut-être surtout, hiérarchie des rôles.

L’expert est donc conçu comme un prestataire de service. Il apporte son concours à la Justice dans les limites qui lui sont signifiées par son commanditaire. L’horizon de l'expert s'arrête à l'énoncé de la mission. «La mission, rien que la mission, mais toute la mission » ${ }^{21}$ se plaît à marteler un éminent juriste, Conseiller honoraire à la Cour de cassation. Or, il serait pour le moins naïf de penser que c'est seulement «l'extériorité du technicien par rapport à l'instance [qui] explique la liberté d'appréciation totale dont jouit le juge, tant à propos du recours au technicien qu'à l'égard des conclusions de ce dernier » ${ }^{22}$. C'est bien plutôt la volonté de limiter le rôle de l'expert dans le procès qui motive un tel état de fait.

\footnotetext{
${ }^{18}$ Michel OLIVIER, « Aspects juridiques et déontologiques du rapport d'expertise vétérinaire », in Michel. OLIVIER, De l'expertise civile et des experts, t. 2, Paris, Berger-Levrault, 1995, p. 40.

${ }^{19}$ Ibid.

${ }^{20}$ Jean PRADEL, « Les rôles respectifs du juge et du technicien dans l'administration de la preuve en matière pénale », in INSTITUTS D'ETUDES JUDICIAIRES, Les rôles respectifs du juge et du technicien dans l'administration de la preuve, Paris, Puf, 1976, p. 68.

${ }^{21}$ Michel OLIVIER, « L'expertise en matière civile », in Michel OLIVIER, De l'expertise civile et des experts, t. 2, op. cit. p. 17.

${ }^{22}$ Bruno OPPETIT, «Les rôles respectifs du juge et du technicien dans l'administration de la preuve en droit privé », INSTITUTS D'ETUDES JUDICIAIRES, Les rôles respectifs du juge et du technicien dans l'administration de la preuve, Paris, Puf, 1976, p. 57.
} 
L’instrumentalisation des experts répond et correspond à une vision sacralisée du droit, de l'institution judiciaire et de ses praticiens. Seuls, les juristes maîtrisent cet art indéfinissable qu'est l'art juridique ${ }^{23}$, seuls les magistrats entretiennent « un lien insondable avec la souveraineté ${ }^{24}$. «Quelle condition, en effet, que celle du Magistrat! De quelle haute mission il se trouve revêtu! Placé par la loi au milieu de la société, comme sur un siège élevé, pour pouvoir mieux découvrir les désordres qui en troublent l'harmonie... ne participe-t-il pas, en quelque sorte, à la puissance souveraine, dont le plus bel apanage, comme le devoir le plus sacré, est de distribuer la justice aux peuples réunis sous ses lois ? Et si ce pouvoir n'est qu'une image, une émanation de celui du créateur lui-même, dont la mission invisible gouverne l'Univers, le magistrat n'est-il pas pour ainsi dire, d'une nature plus qu'humaine ? ${ }^{25}$.

Elevé par les fonctions qu'il accomplit, le magistrat est un homme de vertu, exceptionnel et presque surhumain, directement en contact avec la sacralité et le surnaturel ${ }^{26}$. L'expert, personnage profane immiscé dans le sanctuaire de la Justice, ne saurait déborder le cadre fixé par le magistrat sans risquer d' « altér[er] la pureté de la fonction juridictionnelle » ${ }^{27}$. L’art de juger et la définition du juste doivent demeurer l'apanage du droit, de la Raison juridique et des juristes. La division du travail entre expert et magistrat est donc plus qu'une différence de compétences, c'est une question d’identité et de légitimité. Du point de vue du droit et des juristes, l'expert n’est légitime à intervenir dans le processus judiciaire que dans la mesure où il y a été convié par le tribunal.

\footnotetext{
23 Jacques COMMAILLE, L'esprit sociologique des lois. Essai de sociologie politique du droit, Paris, Puf, coll. « Droit, éthique, société », 1994, p. 221.

${ }^{24}$ François-Xavier TESTU, « Présentation générale », in Guy CANIVET, L'expertise, Paris, Dalloz, p. 7.

${ }^{25}$ G. de ROULHAC, «La dignité de la magistrature en général et de celle de la Cour en particulier », discours de rentrée de la Cour d'appel, 9 novembre 1812, cité par Jean-Claude FARCY, Magistrats en majesté. Les discours de rentrée aux audiences solennelles des cours d'appel (XIXe-XXe siècles), Paris, CNRS Editions, 1998, p.150.

${ }^{26}$ Alain BANCAUD, La haute magistrature judiciaire entre politique et sacerdoce ou le culte des vertus moyennes, Paris, LGDJ, coll. « Droit et société », 1993.

${ }^{27}$ Bruno OPPETIT, op. cit. p. 62.
} 
Comme toutes les relations magistrats-auxiliaires de Justice, la relation d'expertise s’inscrit dans un rapport de domination classiquement institué et mis en œuvre par les juristes ${ }^{28}$. Le juge tire de la loi les moyens d'entretenir avec les experts un rapport de subordination. A travers sa pratique, il actionne et fait résonner le cadre juridique posé par le "législateur". En l’occurrence, si les juristes tolèrent la présence du profane et de l’immanent, c'est seulement à l'intérieur des limites qu'ils leur ont assignées. Convoqués pour gérer la dimension technique et factuelle du litige, les experts doivent s'abstenir de toute immixtion dans les aspects juridiques et décisionnels de l'instance. Sortes de sous-traitants du magistrat, ils prennent en charge la réalisation d'une mission pour laquelle ils suivent un cahier des charges scrupuleusement défini par leur commanditaire. Réservées aux seuls initiés - les "prêtres" de l’ordre juridique - les questions de droit, et en particulier la fonction décisoire, sont interdites aux experts ${ }^{29}$.

Or, ce que la loi proscrit c’est précisément ce qu’elle redoute. C’est bien parce que l'introduction d'une logique non juridique est potentiellement dangereuse et subversive, que les juristes procèdent à son endiguement. Susceptibles de remettre en cause les attributions traditionnelles du juge, d'entrer en concurrence avec le droit et les magistrats dans l'exercice du jugement et plus largement de porter atteinte à l’intégrité et à la validité de la régulation juridique, les savoirs experts sont étroitement encadrés, confinés dans l'espace du secondaire, de l'annexe, de l’accessoire. Sources potentielles de déstabilisation et de concurrence, capables de remettre en cause les principes et les équilibres sur lesquels repose l’édifice judiciaire, les disciplines et acteurs extra juridiques sont instrumentalisés. L’expertise est réduite à sa dimension de procédure et dépouillée de ses enjeux.

\footnotetext{
${ }^{28}$ Ce rapport de domination s’appuie notamment sur les qualités du magistrat : désintéressement, souci du bien public et de l'intérêt général... Citant un courrier émanant d'une Cour d'appel, Jacques Commaille montre que « l'image du magistrat est d'abord construite à partir, a contrario, d'une critique sévère des auxiliaires de justice ». Voir Jacques COMMAILLE, Territoires de justice. Une sociologie politique de la carte judiciaire, Paris, Puf, coll. « Droit et Justice », 2000, p. 53.

${ }^{29}$ Voir l'article $238 \mathrm{du}$ NCPC et son commentaire : «Le technicien n'a pas de pouvoir décisoire. Il ne peut dire le droit, ce qui est le privilège du juge », Michel OLIVIER, " Mesures d’instruction confiées à un technicien », Répertoire de procédure civile, Dalloz, 1997, p. 26.
} 
Le Nouveau code de procédure civile, qui marque un encadrement accru de la procédure d’expertise, peut donc être interprété comme une réaffirmation de la prétention et de la vocation du droit et de ses praticiens à énoncer, mettre en œuvre et protéger les valeurs socialement prégnantes, en particulier, la notion de justice. Ce texte organise et garantit la supériorité du juge et de l’ordre juridique sur les savoirs. Il donne au magistrat les outils juridiques qui lui permettent de durcir leur pratique juridictionnelle et de faire des connaissances, compétences et savoir-faire spécialisés des instruments dans le processus décisionnel. Loin d'être prisonnier de la parole expertale, le magistrat dispose des moyens légaux d'apprécier le rapport d'expertise en toute liberté.

\section{2. "L'art de la pioche"}

De fait, le rapport d’expertise apparaît comme un réservoir d’idées à l’intérieur duquel les juges piochent, isolent un certain nombre d'éléments. En effet, le rapport d'expertise n’est pas envisagé par les acteurs comme un bloc monolithique mais au contraire comme un ensemble composé d'une multiplicité d'unités qui peuvent être dissociées mais aussi réagencées soit entre elles soit avec d'autres items issus des témoignages, des attestations etc. Il fonctionne alors comme une série de monades, chacune d'entre elles pouvant être isolée de son milieu tout en gardant son sens propre. Le démembrement auquel procèdent les acteurs judiciaires (magistrats et avocats) vient à l'appui de leurs stratégies discursives ${ }^{30}$. Chaque acteur invoque tel ou tel passage de l'expertise pour soutenir son argumentation et utilise ainsi les savoirs comme des ressources, c'est-à-dire des «réserves de force ${ }^{31}$. Le rapport d'expertise fonctionne alors comme un pourvoyeur de pièces du dossier, de chiffres, de mots, d'évaluations, d’arguments qui peuvent être empruntés opportunément par les uns et les autres et intégrés dans un raisonnement.

\footnotetext{
${ }^{30}$ Pierre HEBRAUD, « Rapport introductif », in INSTITUT D’ETUDES JUDICIAIRES, La logique judiciaire, Paris, Puf, 1969, p. 33.

${ }^{31}$ L'expression est empruntée à l'encyclopédie Larousse qui donne pour définition du mot « ressources » : « richesses dont on peut disposer : ressources en argent, en hommes, en blé etc. // Moyens d'actions, réserve de forces, d'ingéniosité, etc. : Déployer toutes les ressources de son intelligence », Dictionnaire encyclopédique Larousse, article « Ressource », éd. 1978. C’est bien cette idée de réserve de forces qui nous intéresse, elle colle très bien à ce qu'est le rapport d'expertise tels que le donnent à voir les usages dont il est l'objet.
} 
Le litige suivant qu’a eu à connaître la Chambre de la construction du TGI de Grenoble est particulièrement éloquent ${ }^{32}$. Les requérants se plaignent d'un glissement de terrain ayant emporté une partie de leur terrasse ainsi que le chemin privé situé en contrebas de leur villa. Dans son rapport, l'expert conclut par une imputation des responsabilités de l'ordre de 70 \% pour l'architecte qui a assumé la maîtrise d'œuvre et de 30 \% pour la commune qui, deux ans avant le sinistre, avait fait procéder à la pose de canalisations enterrées au pied du talus de la propriété en question. Le jugement qui reprend les constatations de l'expert mais aussi les causes qu’il avance, s'en défausse en ce qui concerne les responsabilités. Il refuse tout d’abord de se prononcer sur la responsabilité de la commune, dans le mesure où il s'agit d'un acteur public relevant de la seule compétence du Tribunal administratif. Si le juge rejoint ensuite l’expert sur l’idée d’une responsabilité écrasante du maître d’œuvre (80 \%), il impute aux requérants une part de responsabilité (20\%), proposant ainsi un partage de la charge qui n’épargne pas les victimes elles-mêmes. Comment le magistrat a-t-il pu parvenir à ce résultat, assez différent de ce que proposait l'expert ?

Précisément, il a utilisé les documents recueillis par le technicien et annexés dans son rapport. En particulier, il s’est attardé sur une convention, signée entre la commune et les demandeurs, qui stipulait la responsabilité exclusive des maîtres d’ouvrage dans l'élargissement du chemin communal permettant l'accès à leur propriété. On voit bien ici que si l'expert apporte des éléments factuels vérifiés, des enchaînements causaux qui permettent d'entrevoir des responsabilités, le magistrat a la possibilité de s'écarter des interprétations et imputations que l'expert en fait découler. D’autres raisonnements peuvent être développés, argumentés et à cet égard, le choix de privilégier ou d'ignorer une pièce au dossier peut être déterminant. Cette convention passée entre les différentes parties au procès dresse la carte des engagements réciproques et amène ainsi le magistrat à démêler le jeu des responsabilités croisées d’une manière assez différente de celle de l'expert. Cet exemple montre le juge en plein exercice d'un art constitutif de l'art judiciaire, l'art de la pioche.

${ }^{32}$ Dossier B5. 
De même, il arrive que dans un dossier, expertises et contre-expertises se succèdent et se contredisent ; c’est particulièrement le cas lorsque interviennent des experts en assurance. Face à des positions contradictoires et quelquefois partisanes, le tribunal n’est pas forcément dépourvu de moyens d'action. L'exemple qui suit, illustre avec éclat la liberté dont peut user le magistrat à la lecture des rapports ${ }^{33}$.

Dans un contentieux automobile qui oppose une société propriétaire d'un véhicule endommagé et sa compagnie d'assurances, de nombreuses expertises réalisées (une expertise judiciaire et plusieurs expertises officieuses effectuées pour le compte des parties) aboutissent à des thèses éloignées voire divergentes. Le magistrat critique ouvertement la qualité des rapports produits ce qui ne l'empêche pas, un peu plus loin de prendre pour argument le fait que «les constatations des experts, malgré leurs conclusions contradictoires, permettent de retenir qu'il y avait plusieurs traces de chocs sans qu'aucun d'eux n'établisse sérieusement que le passage à vitesse réduite sur ce ralentisseur pouvait entraîner le dommage majeur ${ }^{34}$. Néanmoins, troublé par les conclusions contradictoires des experts, le magistrat considère que le demandeur «n'apporte pas de preuve suffisante pour établir que le dommage qu'il invoque se serait produit comme il l'affirme ${ }^{35}$. Conscient d'avoir affaire à des avis intéressés proposant des interprétations douteuses voire fantaisistes et en tout cas non démontrées, il tire ce qu’il peut des éléments consensuels et en particulier des constatations effectuées. S’émancipant autant que possible des affirmations partisanes, il réintègre les éléments piochés, dans son argumentation globale, introduisant pour finir un argument juridique décisif : le demandeur doit apporter la preuve de ce qu'il avance.

Mais les magistrats n’ont pas le monopole de l'art de la pioche. Les avocats pratiquent eux aussi le démembrement des savoirs et leur instrumentalisation dans le cadre de stratégies

\footnotetext{
33 Dossier A1.

34 Jugement du TGI, 13 février 1997, p. 5.

${ }^{35}$ Ibid.
} 
discursives. Ainsi, dans un litige de la construction ${ }^{36}$, l'avocat de la principale société incriminée critique ouvertement le rapport d'expertise, qualifiant les propos de l'expert de « supputations ${ }^{37}$. Cela ne l'empêche pas de reprendre ensuite certaines préconisations de l'expert qu'il vient pourtant de dénoncer. Incontestablement, l'avocat évalue la qualité de l'expertise à l'aune de sa compatibilité avec les intérêt de son propre client. Lorsque l'expert avance une explication des désordres qui dessert sa partie, le conseil disqualifie l'expertise et / ou l'expert brutalement. En revanche, lorsque l'avis du technicien va dans le sens des intérêts qu’il représente, l'avocat le qualifie de «juste ${ }^{38}$, précisément parce qu’il lui est favorable.

Il arrive aussi que les conclusions expertales soient entièrement favorables à sa partie. L'avocat les reprend alors intégralement et fonde l'ensemble de ses conclusions sur cet unique support argumentatif. Dans une affaire de construction, un des avocats appuie ainsi toute son argumentation sur les acquis de l'expertise ${ }^{39}$. Il en commente les résultats, cite des passages du rapport, et termine par cette phrase : «tout est dit dans ces conclusions » ${ }^{40}$. Il exploite et utilise à plein un avis expertal vu comme salutaire.

L’expertise est donc appréhendée à travers sa capacité d’adéquation au récit de l’avocat. Elle est lue à travers le prisme de la stratégie défensive ou offensive de celui-ci. La grille de lecture actionnée est bien celle de l’opportunité de la parole expertale. Bien que les pratiques des magistrats et des avocats servent des objectifs distincts, en matière d'expertise, elles procèdent selon le même canevas. Conformément au discours développé dans les dispositifs juridiques formels, les praticiens du droit envisagent le rapport d'expertise comme une ressource stratégique dans un processus argumentatif.

\footnotetext{
${ }^{36}$ Dossier B4.

${ }^{37}$ Conclusions, 10 novembre 1994, p. 6.

${ }^{38}$ Conclusions additionnelles, 6 mai 1996, p. 2.

${ }^{39}$ Dossier B4.

${ }^{40}$ Conclusions au fond après expertise, 30 mars 1987, p. 4.
} 


\subsection{Le rapport d'expertise, ressource stratégique ${ }^{41}$}

Les avocats ont pour objectif de gagner le procès c’est-à-dire de faire que ce qui n’était que leur version des faits ou celle de leur client, en lutte avec d'autres interprétations soit validée par le jugement ; qu’elle devienne non seulement la version dominante et officialisée mais plus encore la réalité, en vertu du principe de l'autorité de la chose jugée. "[L]e rôle de l'avocat n'est pas d'établir une vérité certaine, une vérité démontrée, une vérité irréfutable. Il n'est que d'obtenir l'adhésion momentanée des juges, exprimée dans un jugement qui énonce une vérité judiciaire suffisante à la satisfaction de l'avocat ${ }^{42}$. De ce point de vue, les avocats développent une stratégie, entendue à la fois au sens militaire ${ }^{43}$ et au sens que Michel de Certeau lui a donné dans L'invention du quotidien ${ }^{44}$. Il oppose en effet la stratégie à la tactique, à la ruse, qui ne se développe pas à partir d'un espace propre mais à l'intérieur même de la domination qu'elle ne peut contester ouvertement : la tactique c’est la résistance opérée depuis l’intérieur.

La préoccupation et l'action des avocats sont bien d’ordre stratégique. Le conseil s’identifie à celui qu'il représente au point de parler en son nom et à sa place, d'utiliser la première personne du pluriel qui indique, sinon la cohésion, du moins le partage d'une situation. Il y a là un lieu propre, une singularité qui se distingue très nettement voire très violemment de l'autre, l'ennemi, l'adversaire, le "contradicteur", avec lequel le rapport de force s'engage de manière frontale et physique (audiences, reconstitutions, transports sur les lieux...) ou indirecte et médiatisée (courrier, dépositions, attestations...).

\footnotetext{
${ }^{41}$ Précisons qu'il est ici question de l'utilisation stratégique du rapport d'expertise. En effet, le format de cet article ne permet pas de traiter des jeux que suscite la mise en œuvre de la procédure d'expertise.

42 Jean-Denis BREDIN, « La logique judiciaire et l'avocat », in INSTITUT D’ETUDES JUDICIAIRES, op. cit. p. 94.

43 «Art de coordonner des actions et de manœuvrer pour atteindre un but», dictionnaire encyclopédique Larousse, article « Ressource », op. cit.

${ }^{44}$ «J'appelle stratégie, le calcul (ou la manipulation) des rapports de force qui devient possible à partir du moment où un sujet de vouloir et de pouvoir (une entreprise, une armée, une cité, une institution scientifique) est isolable. Elle postule un lieu susceptible d'être circonscrit comme un propre et d'être la base d'où gérer les relations avec une extériorité de cibles ou de menaces (les clients ou les concurrents, les ennemis, la campagne autour de la ville, les objectifs et objets de recherche, etc.) », Michel de CERTEAU, L'invention du quotidien, t. 1, Paris, Gallimard, coll. « Folio Essais », 1990, p. 59.
} 
Quant aux magistrats, la notion de stratégie rend compte, là aussi, d’un certain aspect du travail judiciaire. En particulier, elle traduit bien l'idée d’un juge porteur d'une position propre par rapport au litige, position à la fois professionnelle et personnelle, issue de la confrontation des données du dossier avec ses références et représentations, qu’elles soient issues de son expérience individuelle, de sa formation initiale, de son appartenance à un groupement syndical ou encore de considérations générales émises par la juridiction dans laquelle il exerce ou par la Chancellerie dans le cadre de la formulation de politiques pénales globales. La dimension stratégique permet d’écarter définitivement la figure du magistrat "bouche de la loi", cette fiction juridique qui enferme les praticiens du droit dans une nécessaire passivité et occulte la complexité du processus de construction du jugement, lui-même réduit, via la fiction du raisonnement syllogistique, à un procédé mécanique d’application de normes générales à des cas particuliers.

A l'évidence, le magistrat jouit d’une liberté d’action dans la résolution des contentieux. Evoluant dans un jeu relativement ouvert, il arbitre entre une pluralité de solutions hétérogènes et non équivalentes, mais également susceptibles de mettre un terme au conflit. Le litige de la construction qui suit ${ }^{45}$ donne à voir la formulation par le juge, d'une position propre, la mise en œuvre d’une stratégie subjectivement déterminée et résultant d’un choix opéré entre plusieurs alternatives.

Dans un contentieux entre les propriétaires d'un appartement neuf et les entreprises qui ont procédé à sa construction, bien que l'expert atteste de la réalité des désordres allégués par les demandeurs, qu'il identifie avec certitude l’origine et l'entreprise responsable, le magistrat déboute les requérants. Il considère en effet que les dérèglements constatés n’entrent pas dans le cadre de la garantie décennale. Il justifie alors sa position par une interprétation "serrée" de la loi, alors qu’il ne tenait qu’à lui, justement, de procéder à une lecture plus souple des textes. S’agissant de défauts sur un revêtement de marbre, il aurait pu considérer que le marbre étant un revêtement de prestige,

\footnotetext{
${ }^{45}$ Dossier B1.
} 
présenté et vendu à ce titre aux demandeurs, ses défauts esthétiques le rendaient impropre à sa destination et entraient de ce fait dans le champ de la garantie décennale.

Or, le motif invoqué - en l’occurrence les lacunes de la loi - relève bien d'un procédé argumentatif et stratégique. Ni impérative, ni incontournable, cette lecture des textes constitue l'exercice d'un choix et d'une liberté. Le règlement du litige et la définition de la meilleure solution sont étroitement liés à la manière dont est appréciée la situation : est-elle plus ou moins grave, plus ou moins scandaleuse...? Bien que parfaitement justifiées, les doléances des demandeurs sont apparues, aux yeux du magistrat, secondaires et relatives. Le fait que celui-ci considère comme superflu et somptuaire le fait de vivre dans un appartement dallé de marbre a certainement influé sur sa manière de dire le droit.

Acteur disposant certes d'un capital d'autorité singulier, le magistrat n’est ni une puissance arbitrale qui serait placée en terrain neutre, ni une puissance transcendante qui trônerait au-dessus du litige et des parties, c’est un acteur immergé "dans la mêlée", confronté à des choix qu’il réalise en s'appuyant sur ses connaissances juridiques, en examinant rationnellement la situation mais aussi en se fiant à ses intuitions, en faisant jouer son bon sens, en reproduisant des habitudes... En somme, le jugement s'élabore au fil d'un processus cognitif complexe, que les motivations rationalisent et justifient a posteriori ${ }^{46}$. Dans le cadre de ce processus, le rapport d'expertise joue le rôle d'un outil utile pour la formulation des solutions mais aussi pour leur justification. Cet instrument peut être massivement utilisé au point de devenir le socle unique sur lequel se fonde le jugement. Ressource exclusive du juge, le rapport d'expertise est homologué sans l'ombre d'une critique, il est même protégé des attaques partisanes.

\subsection{Le rapport d'expertise, ressource exclusive}

Il arrive de rencontrer des dossiers dans lesquels le magistrat renonce d'emblée à toute discussion, à toute position critique par rapport aux résultats de l'expertise. Alors qu'il pourrait 
avoir prise sur le rapport, qu'il pourrait interroger certains raisonnements, certains chiffrages, le juge entérine purement et simplement la parole expertale et fait d'elle la source constitutive du jugement. Il s’abstient de discuter ou d’évaluer les affirmations du technicien, certainement plus par habitude que par principe. En effet, ce cas de figure correspond le plus souvent à des dossiers de routine - le "pain quotidien" de la Justice - relatifs à des litiges répétitifs et stéréotypés autour desquels pointe une certaine routinisation du métier de magistrat. Les contentieux de loyers commerciaux sont exemplaires de ce point de vue. Le libellé et le contenu de la mission varient peu et s'inscrivent dans une configuration stable d'un procès à l'autre : deux parties, deux positions clairement identifiées, deux revendications très polarisées ${ }^{47}$, des arguments comparables voire identiques, en particulier lorsqu'il s'agit de locaux situés dans le même contexte géographique ${ }^{48}$. C’est également le cas de certains litiges de la famille, relatifs au divorce et à l'hébergement des enfants en particulier. Toujours originaux et contextualisés, ces différends répondent néanmoins à des types assez stables : chaque parent réclame l'hébergement principal des enfants ou l'exercice d'un droit de visite le plus souple possible.

Le magistrat n’hésite pas à reprendre intégralement les conclusions avancées dans le rapport et à les retranscrire telles quelles dans les attendus du jugement. Plusieurs affaires témoignent de cette manière de faire, partagée par plusieurs juges mais inégalement mise en œuvre. Dans un litige qui oppose le bailleur et le locataire d'un hôtel de quartier ${ }^{49}$, le tribunal affirme, après avoir repris les arguments de l'expert relatifs au choix d'un mode de calcul spécifique, «l'évaluation de la valeur locative arrêtée par l'expert sur le fondement de l'article 23-8 n'est pas discutée dans sa

\footnotetext{
${ }^{46}$ Voir notamment Giandomenico MAJONE, Evidence, Argument and Persuasion in the Policy Process, New Haven / Londres, Yale University Press, 1989, en particulier p. 29-30.

${ }^{47}$ Les parties argumentent dans deux directions opposées : les facteurs locaux de commercialité ont été modifiés durant le bail écoulé ce qui justifie un déplafonnement et une fixation du loyer en fonction de la valeur locative (position du bailleur) versus il n'y a pas eu de modification notable de ces facteurs locaux de commercialité, ce qui implique que le bail soit renouvelé sans déplafonnement (position du preneur).

${ }^{48}$ La fixation de loyers commerciaux à Grenoble à une époque donnée repose ainsi sur des arguments identiques : installation du tram, piétonnisation, construction du pôle technologique Europole, etc.

${ }^{49}$ Dossier C2.
} 
mise en œuvre par les parties, et elle n'appelle effectivement pas de critiques. Il convient de dire que le loyer du bail renouvelé le 1er février 1994 doit être fixé à $71594 \mathrm{~F} »^{50}$.

De même, dans un autre dossier, tandis que les avocats contestent le rapport, le magistrat reprend l'intégralité des conclusions expertales ${ }^{51}$. Il se déclare ouvertement solidaire de celui qu'il a désigné : «le juge des loyers commerciaux ne saurait en la circonstance avoir d'autre opinion que celle de M. X, qu'il a désigné en qualité d'expert (...) au terme d'un rapport que le juge estime devoir faire sien ${ }^{52}$. Il justifie alors sa position par des arguments d'autorité relatifs au statut de l'expert. En effet, tout se passe comme si le simple fait que l'expert soit missionné par le juge garantissait l'intérêt et la qualité de l'intervention sans même que le magistrat n'interroge ou ne fasse état de la qualité effective de l’expert (diplômes, expérience, etc.) ou du rapport d’expertise (clarté, impartialité, précision, etc.). Face aux attaques et dénigrement des avocats, le magistrat plutôt que d'argumenter sur la qualité intrinsèque du technicien ou sur la pertinence de ses calculs et considérations - étend son aile protectrice sur le rapport d'expertise. C’est ainsi la continuité entre le juge et l'expert qui est soulignée. L’arbitraire du premier s’exerce pour "couvrir" la parole du second dont il reprend mot pour mot et chiffre pour chiffre les conclusions. Cette reprise se fait naturellement dans la mesure où l'expert est en quelque sorte l'œil et l'oreille du magistrat, son "correspondant" sur les lieux.

Conception de l'expertise mais aussi relations magistrat-expert, routinisation des conflits, manque de temps et nécessité de faire vite peuvent être invoqués pour expliquer cette utilisation "totale" et systématique de l’expertise. Cette procédure contribue alors à un traitement accéléré des dossiers, particulièrement utile dans le cadre d'une Justice confrontée à une augmentation massive et durable du contentieux. Quoi qu'il en soit, le magistrat limite ici volontairement sa liberté d'action. En validant l'expertise, il renonce à exercer son pouvoir d'arbitrage. Toujours acteur de la

\footnotetext{
50 Jugement du TGI, 3 février 1997, p. 5.

${ }^{51}$ Dossier C1.

52 Jugement du TGI, 27 octobre 1997, p. 2.
} 
décision et détenteur officiel du pouvoir de décider, il se place dans une position quasi administrative de ratification d'une solution qui lui est fournie "clef en main".

Cependant, la valorisation des résultats d'expertise dans le jugement ne repose pas exclusivement sur le bon vouloir du magistrat. En ce sens, le rapport d'expertise ne représente pas seulement un "moyen" pour les acteurs judiciaires d'atteindre leurs objectifs de persuasion. La dimension de ressource est indissociable de celle de contrainte. Si dans un cas, le juge décide et choisit ce qu'il retient de l'expertise - y compris tout au point d'abdiquer son propre pouvoir -, dans un autre, il est limité dans sa capacité de décision par les résultats du rapport d’expertise. Dans des cas extrêmes, la portée de la parole expertale est telle que se dessine pour le magistrat un rôle mineur : celui qui consiste à décider des conséquences juridiques provoquées par les résultats scientifiques énoncés au cours de l'expertise.

\section{L'expertise judiciaire : une contrainte imposée}

Si la doctrine et les dispositifs formels tentent effectivement de réduire l'expertise à une ressource parmi d'autres, à un outil dans le cadre de la construction du jugement, l'observation des dossiers judiciaires montre qu'elle est bien plus qu'un procédé de production et de distillation d'informations que le magistrat et l'avocat utiliseraient à leur guise. La conception décisionniste du rapport entre savoir et pouvoir semble de ce point de vue partielle, limitée et restrictive. Elle ignore d’emblée la capacité contraignante des savoirs et savoir-faire qu'elle aborde comme des accessoires. Or, l'introduction de la technicité non juridique dans le champ judiciaire est porteuse d'effets sur le système décisionnel. Non seulement elle bouleverse l'équilibre des ressources disponibles mais encore provoque-t-elle l'apparition d'une force de coercition qui s'impose au magistrat. Les savoirs ont en effet la capacité de peser sur l'élaboration du jugement, au moins dans certaines configurations. Le rapport d’expertise enferme doublement le magistrat : récit constitutif du réel, il est aussi discours explicatif du réel. 


\subsection{Le rapport d'expertise, récit constitutif du réel}

Le rapport d'expertise est un exercice particulier qui répond sinon à des règles académiques strictes, du moins à des habitudes installées. Traditionnellement, le plan s’organise autour de quatre parties distinctes : le préambule, l'exposé des opérations d’expertise et des constatations effectuées, la discussion des faits établis et enfin l'avis c'est-à-dire la présentation synthétique des conclusions auquel le technicien aboutit ${ }^{53}$. Ce découpage en quatre temps représente plus qu'une matrice fonctionnelle : il exprime la profonde hétérogénéité de la parole expertale.

Dans les deux premières parties, l'exercice est de type analytique et synthétique. Le préambule contient le rappel des termes de la mission, le résumé du contexte procédural et la présentation des parties à l'instance, de leurs positions et prétentions réciproques. Dans la partie consacrée aux opérations, l’expert procède au récit du déroulement de sa mission : chronologie des réunions, nature des actes pratiqués, résultats des observations, réactions des parties sont mises à plat. L'auteur du rapport narre ainsi à son commanditaire les événements intervenus pendant l’expertise. Il restitue le produit brut de ses déplacements sur le terrain, la matière première de son compte-rendu : observations, constats, cotes, relevés... autour de l’objet ou du sujet expertisé et des désordres qui l'affectent. La description physique des lieux ou des personnes, la mesure et le pesage, la prise de photos constituent autant de manières de restituer une certaine réalité et de la rendre accessible au magistrat.

La description n'est pas seulement restitution, elle est aussi institution. L'expert fait son rapport au sens où il rend compte, de ce qu'il a vu et entendu sur le terrain mais c'est justement sur la base - et sur la foi - de cet état des lieux que seront établies la réalité et la vérité judiciaires. La parole expertale en racontant le réel contribue précisément à le façonner et ce, du fait de son statut original dans l'instance. Statut doublement original en effet que celui du discours expertal. Parole dotée d'un certain pouvoir d'ordonnancement du réel, parce que fondée sur une légitimité de nature

\footnotetext{
${ }^{53}$ Michel OLIVIER, « Rédaction du rapport d'expertise en matière civile », in Michel OLIVIER, De l'expertise civile et des experts, t. 2, op. cit. p. 49-55, voir également du même auteur et dans le même volume, "Modèle du rapport d'exécution d'une mesure d'instruction en matière judiciaire civile », p. 57-64.
} 
scientifique, elle tire également cette présomption de vérité d’une incontestable caution juridique et judiciaire. Le mandatement par le magistrat, assorti de la prestation de serment confère un statut spécifique aux propos de l’homme de l'art. A nouveau, c'est toute la respectabilité de l'institution et de ses officiants qui rejaillit sur celui qu’elle désigne et dont elle garantit plus que la compétence : l’absence d’intérêts personnels et l'extériorité par rapport au conflit. L’expert incarne alors l'homme de confiance du magistrat, le narrateur fidèle et intègre qui donne à voir une réalité objectivée et attestée.

Cette exceptionnalité de la parole expertale est fondée et exprimée par le statut juridique spécifique reconnu au rapport d'expertise. Celui-ci est en effet «revêtu tout comme l'acte authentique, de la force probante absolue ${ }^{54}$. Le discours expert est alors assimilé aux actes certifiés et authentifiés. Comme eux, il est constitutif de faits établis qui ne pourront être contestés, sauf inscription en faux. Au fil de l'évolution de la jurisprudence, le préambule, l'exposé du déroulement de la mission et la description des constatations ont conservé leur force probante absolue, et ce, même si le Nouveau code de procédure civile pose en son article 246 que « le juge n'est pas lié par les constatations ou les conclusions du technicien ». En revanche, les autres parties du rapport - la discussion et les conclusions - sont désormais «soumises à la simple preuve contraire $»^{55}$.

En somme, malgré un relatif déclin de sa force probante, le statut du discours expert reste spécifique et fonde le rôle original de cette parole dans le processus judiciaire. Le rapport d’expertise en construisant un double récit, participe de la constitution du réel. Il établit l’histoire de l’expertise, raconte comment elle s’est passée, à quels échanges elle a donné lieu. Mais plus encore, ce compte-rendu construit la réalité et l’objet du litige, la nature des désordres en cause. A

\footnotetext{
${ }^{54}$ Michel OLIVIER, « L'expertise en matière civile », in Michel OLIVIER, De l'expertise civile et des experts, t. 2, op. cit. p. 20.

${ }^{55}$ Ibid.
} 
l'attention du magistrat mais aussi des parties et de leurs conseils, il dresse le diagnostic de la situation, la carte des douleurs, dysfonctionnements et pannes.

Le technicien appréhende la situation à partir de la mise en éveil de ses cinq sens. Il observe un quartier, scrute des blessures, passe au crible une œuvre d'art, regarde en détail l'état d'une construction ou d'une automobile, remarque les détails physiques et comportementaux révélateurs du patient, recherche des traces perceptibles à l'œil nu ou avec un instrument optique. Il touche, palpe et tâte le corps de son patient, passe la main sur l'enduit. Il respire le parfum d'un vin, détecte les odeurs de produit inflammable, renifle la puanteur des canalisations défectueuses. Il prête l'oreille aux bruits anormaux, écoute les doléances de chacun, entend les remarques des uns et des autres. Il met en marche ses papilles gustatives, goûte, déguste et apprécie la qualité d’une boisson ou d'un met. L’expert recourt à l'exercice des sens, à l'expérience et l'expérimentation sensibles. Mais cette première confrontation n’est pas pur éveil des sens : elle est nourrie de connaissances théoriques, d'expériences et de savoir-faire de même qu'elle peut prendre la forme d'une quantification par l'utilisation d'appareils ou de systèmes de mesure, l'application de méthodes d'analyse, de calcul etc.

L'expert rend compte de sa perception directe ou indirecte dans son rapport et ses comptesrendus de réunions. Le ton est alors celui de la description et de l'affirmation. Le temps utilisé est soit le présent de l’indicatif soit le passé composé qui, tous deux véhiculent l’idée d'un état de fait et insistent sur la certitude de celui qui l'affirme. Le vocabulaire appartient au champ sémantique de la constatation et de l'observation : « après examen attentif de la partie inférieure du carter, nous avons relevé des traces de rayures suite à un accrochage antérieur » ${ }^{56}$; "la baignoire a été réparée avec de l'émail à froid. Cette réparation est très peu visible ${ }^{57}$; “la marche s'effectue sans boiterie. La marche sur la pointe des pieds ou le talons est possible et indolore. (...) Il n'y a

\footnotetext{
${ }^{56}$ Dossier A1, rapport d'expertise,25 janvier 1994, p. 2.

${ }^{57}$ Dossier B4, rapport d'expertise, 9 décembre 1985, p. 15.
} 
pas de bascule du bassin. (...) L'abduction est de $35^{\circ}$ à droite et $40^{\circ}$ à gauche ${ }^{58}$. Ces phrases courtes et simples, de style direct, dressent des constatations, elles recensent et répertorient des désordres qui, du fait qu’ils sont inventoriés, entrent dans l'espace judiciaire et deviennent une réalité prise en compte dans le jugement. Par un effet de miroir, les faits qui ne sont pas retenus par l'expert sont écartés, gommés, oubliés. Ce que l'expert n’a pas vu, n’existe pas ; ce qu’il n’a pas jugé pertinent de prendre en compte perd toute consistance et toute existence.

Ainsi dans un litige de la construction qui oppose maîtres d’ouvrage, maîtres d'œuvre et sous-traitants ${ }^{59}$, l'expert doit «décrire les malfaçons ou non conformités ou inachèvements allégués ${ }^{60}$ qui affectent une usine récemment édifiée. L’Inspection du travail a de son coté relevé un défaut de conception, contraire au Code du travail. Or, l'expert rapporte cette position mais considère que ce vice «ne [lui] semble pas évident» et conclut donc «nous ne le retiendrons pas ${ }^{61}$, écartant ainsi cette réclamation des désordres constatés et enregistrés comme tels, dans le cadre de la procédure judiciaire.

De même, dans un dossier d'accident automobile ${ }^{62}$, le blessé se plaint d'une entorse au poignet droit provoquant «une douleur importante et persistante » ${ }^{63}$ ainsi qu'un handicap important, alors que le médecin expert évoque dans son rapport un examen "relativement pauvre ${ }^{64}$, au terme duquel «les amplitudes de mobilisation active et passive de flexion, extension et inclinaison de la main sur le poignet droit se révèlent strictement normales » ${ }^{65}$. Le médecin dresse le tableau d'une blessure peu grave dans ses manifestations cliniques et dans ses

\footnotetext{
${ }^{58}$ Dossier F2, rapport d'expertise, 28 mars 1995, p. 6-7.

${ }^{59}$ Dossier B6.

${ }^{60}$ Rapport d'expertise, 15 octobre 1993, p.3.

${ }^{61}$ Ibid. p. 5.23 .

${ }^{62}$ Dossier F6.

${ }^{63}$ Assignation devant le Tribunal correctionnel, 6 mai 1997, p. 4.

${ }^{64}$ Rapport d'expertise, 19 janvier 1996, p. 3.

${ }^{65}$ Ibid. p. 3.
} 
conséquences : le patient "présente des séquelles excessivement modestes » ${ }^{66}$ affirme-t-il, ce que reprendra explicitement le magistrat dans le jugement. A n'en pas douter, c'est bien à l'expert qu'il revient de définir la nature de la lésion, d’apprécier la gravité des séquelles, de déterminer les souffrances engendrées et ce, au regard de critères médicaux généraux. Le point de vue médical vient fixer et durcir les dommages corporels qui seront soumis à réparation financière. La victime fait état de son vécu de l'accident, de ses doléances mais c'est à l'expert qu'il appartient de dire et de fixer la réalité du dommage - sauf à être infirmé par le magistrat.

Ces exemples donnent à voir l'expert en pleine œuvre d'établissement des faits et de discrimination des allégations non fondées. Il dit quels sont les désordres, décide de la pertinence et de la véracité des réclamations respectives et fait la part des choses entre des affirmations contradictoires. Il joue pleinement un rôle de construction et d’attestation de la réalité. Au total, la parole de l'expert est bien une parole instituante qui a le pouvoir de constituer la réalité sur laquelle le magistrat travaille. C’est également une parole explicative qui met en œuvre des démonstrations, des schémas causaux capables d’éclairer le réel, de le rendre compréhensible. A nouveau, l’expertise fonctionne comme discours de vérité ${ }^{67}$ qui s’impose aux acteurs judiciaires.

\subsection{Le rapport d'expertise, récit explicatif du réel}

La codification instaure, dans quelques cas, une contrainte à pratiquer l'expertise ${ }^{68}$. Il arrive que cette contrainte se prolonge avec le rapport d'expertise : celui-ci apporte une réponse incontestable, une certitude inégalable dans l'affirmation de l'impossibilité ou de la réalité d'un événement. Cette configuration se rencontre particulièrement dans les litiges de contestation de filiation. Ces conflits relatifs à une éventuelle inadéquation entre paternité biologique, paternité

\footnotetext{
${ }^{66}$ Ibid. p. 3.

67 Sur le discours de vérité comme ressource dans l’art de gouverner, voir Michel FOUCAULT, «La gouvernementalité », in Michel FOUCAULT, Dits et écrits, 1954-1988, t. 3, n²39, Paris, Gallimard, coll. « Bibliothèque des sciences humaines », 1994, p. 635-657.

${ }^{68}$ C'est le cas notamment de la rescision pour lésion en matière de vente d'immeuble et de la réparation des dommages causés aux récoltes par le gibier, voir Michel OLIVIER, « Mesures d’instruction confiées à un technicien », op. cit. p. 8.
} 
officielle (civile) et paternité sociale (éducative), conduisent à l'utilisation de méthodes identificatoires telles que l’analyse hématologique et l'étude des empreintes génétiques, qui ont la particularité de pouvoir dire avec précision si les individus entretiennent un lien biologique, si ce lien est probable ou s’il est formellement exclu.

Dans un dossier traité par la chambre de la famille ${ }^{69}$ qui oppose deux pères présomptifs mais dont l'un des deux a reconnu l'enfant, le magistrat ordonne une expertise avec pour mission « d'effectuer un examen comparé [du sang prélevé] et de rechercher si en fonction des données actuelles de la science, M. X peut ne pas être le père de l'enfant " ${ }^{70}$. Après un exposé de la méthode utilisée (lymphocytotoxicité) et une interprétation des résultats obtenus, le rapport conclut que «l'étude des génotypes érythrocytaires et leucoplaquettaires HLA ont montré que M. X n’est pas exclu comme père biologique de l'enfant. Le calcul de probabilité “ a posteriori ” établi selon la formule de Bayes permet de montrer qu'[il] a 99,48 chances sur cent d'être le père biologique de l'enfant présenté »" ${ }^{71}$.

Face à de tels résultats, la position des avocats consiste à prendre acte de ces conclusions et à les considérer ipso facto comme des faits établis, et ce , quelle que soit la demande initiale du client. Ainsi, le conseil du demandeur se résigne, indiquant que son client « ne peut que, en application de l'article 338 du Code civil, s'incliner compte tenu du caractère péremptoire de l'analyse hématologique et de la demande reconventionnelle [de son contradicteur]» ${ }^{72}$. Face à cet aplanissement du litige, le rôle du juge se borne à tirer les conséquences des résultats expertaux : « au vu des conclusions du rapport d'expertise le Tribunal ne peut que constater que M. X est bien

\footnotetext{
${ }^{69}$ Dossier D3.

${ }^{70}$ Rapport d’expertise, 6 juin 1995.

${ }^{71}$ Ibid.

${ }^{72}$ Conclusions après expertise, audience du 20 juin 1995.
} 
le père biologique de Laurent. Il convient d'ordonner que cet enfant portera désormais le nom de son père : $X$; que cette mention en soit faite sur les registres de l'Etat civil » ${ }^{73}$.

De même, dans un autre litige relatif au lien de filiation ${ }^{74}$, les conclusions de l'expertise sont là encore formelles. La personne qui avait reconnu l'enfant « est exclu[e] comme père biologique au vu des résultats obtenus par trois systèmes informatifs HLA et les systèmes érythrocytaires Kidd et Duffy ${ }^{75}$. Du coup, l'avocat de la partie adverse fait largement écho aux conclusions expertales : cet individu « est exclu comme père biologique de l'enfant au vu des résultats obtenus par trois systèmes informatifs. [...] Par conséquent, il est établi que la reconnaissance effectuée par [lui] au bénéfice de l'enfant Michel est une reconnaissance de complaisance parfaitement contraire à la réalité. Le Tribunal devra donc annuler cette reconnaissance ${ }^{76}$. C’est ce que ne manque pas de faire le magistrat : «le rapport d'expertise sanguine diligentée par l'expert exclut de façon formelle la paternité de M. X. Dès lors, la reconnaissance de l'enfant Michel qu'il avait souscrite devant l'officier d'Etat civil de la commune M, le 6 mai 1994 doit être annulée ${ }^{77}$.

Dans ces deux cas, les conclusions expertales participent directement de l'établissement des faits et plus encore de la solution du litige. Dès lors que les résultats de l'expertise sont connus, le conflit s'éteint de lui-même. Les avocats prennent acte des affirmations du rapport et les considèrent d'emblée comme des faits acquis au débat. Conséquemment, ils reformulent leur stratégie à l'aune de ces faits nouveaux : soit ils renoncent à argumenter pour une cause perdue soit ils tirent partie de la situation et formulent une demande reconventionnelle par exemple. Quant au juge, il n’a plus qu’à tirer les conséquences juridiques des conclusions expertales, recouvrant son pouvoir décisionnel à la marge, en ce qui concerne les demandes reconventionnelles.

\footnotetext{
${ }^{73}$ Jugement du TGI, 19 juin 1996.

${ }^{74}$ Dossier D1.

${ }^{75}$ Rapport d'expertise, 27 février 1995.

${ }^{76}$ Conclusions après expertise, ss date.

${ }^{77}$ Jugement du TGI, 24 janvier 1996.
} 
Cette configuration spécifique n’a pas été rencontrée dans d’autres types de litiges que la contestation de paternité ou concernant d'autres disciplines d'expertise que l'analyse hématologique. Pourtant, on peut penser que ce pouvoir de contrainte de l'expertise, révélé dans ces cas spécifiques, s'exerce dans d'autres domaines, en matière correctionnelle en particulier, et à partir d’autres savoirs comme la médecine légale par exemple. Les résultats des études ADN semblent être considérés comme peu contestables et jouer le même rôle d'établissement des faits que les analyses de sang.

Ainsi, on peut dire que c'est la capacité d’une discipline à établir des certitudes qui détermine en partie son pouvoir de contrainte sur le réel et en particulier sur le jugement. Précisément, le caractère péremptoire des résultats expertaux est étroitement lié à la nature du savoir mis en œuvre : science exacte, discipline expérimentale, savoir positiviste produisent des énoncés affirmatifs qui sont considérés comme vrais.

Pour autant, il ne s’agit pas de présenter les sciences exactes comme des sciences objectives, qui procéderaient de vérités révélées une fois pour toutes. Les sciences dures comme les sciences sociales et humaines sont des activités sociales qui s’inscrivent dans des configurations sociohistoriques précises ${ }^{78}$. Elles n’offrent pas de vérités absolues mais des vérités provisoires toujours susceptibles d'être réévaluées et contredites plus tard ou ailleurs ${ }^{79}$. Les nombreux débats autour des propriétés de l'arsenic ont bien montré quelle pouvait être la fragilité et la réversibilité des connaissances de chimie légale par exemple ${ }^{80}$.

Les résultats et découvertes des sciences exactes (biologie, physique, chimie...) n’en demeurent pas moins nimbés d’une aura et d’une valeur particulières, ne serait-ce que dans les

\footnotetext{
${ }^{78}$ Voir notamment Bruno LATOUR et Steve WOOLGAR, La vie de laboratoire. La production des faits scientifiques, Paris, La Découverte, coll. « Poche Sciences humaines et sociales », 1996.

${ }^{79}$ Voir Thomas S. KUHN, La structure des révolutions scientifiques, Paris, Flammarion, coll. « Nouvelle bibliothèque scientifique », 1972 et Alan F. CHALMERS, Qu'est-ce que la science ?, Paris, La Découverte, coll. « Biblio essais », 1987.
} 
représentations sociales. Les sciences dures sont vues comme des discours de vérité sur le monde, suscitant une confiance particulière alors que les sciences humaines provoquent un certain scepticisme. Ainsi, certains magistrats établissent d'emblée une distinction entre sciences dures, objectives et fiables, et sciences humaines, subjectives et relativement aléatoires. En témoigne cet extrait d'entretien avec un juge d'instruction : «les expertises qui portent sur des techniques de sciences exactes par exemple une autopsie médico-légale, un taux d'alcoolémie, on ne va pas discuter. C'est vrai qu'on peut discuter mais le taux d'alcoolémie, l'expertise médico-légale en matière d'autopsie, les empreintes génétiques..., on nous dit «c'est comme ça, il n’y a pas à en discuter » [...] Par contre, il y a le domaine où il s'agit de sciences inexactes, la psychiatre, la psychologie, tout ces domaines-là. C'est vrai que c'est un domaine qui est par définition contestable [...]. On est dans un domaine où tout se discute et tout doit d'ailleurs se discuter et c'est clair qu'une expertise psychologique, ce n'est pas une preuve et ça n'en sera jamais une » ${ }^{81}$. A n’en pas douter, cette représentation duale des savoirs scientifiques joue un rôle dans la réception des conclusions expertales : certains résultats sont considérés d’emblée comme constitutifs de preuves irréfragables, incontestables, tandis que d'autres sont appréhendés avec une certaine prudence. Lorsque l'expert affirme qu’il est génétiquement impossible qu’un individu soit le père d'un enfant, les acteurs judiciaires considèrent que c’est une réalité, un fait tangible qui est énoncé. Par ce recours au savoir scientifique, la Justice prétend au vrai, défini et établi non plus par le droit mais par la science.

En somme, le rapport d'expertise doit être envisagé dans sa double dimension de contrainte et de ressource, ce qui permet de concevoir, de restituer et de comprendre la diversité des usages dont il est l'objet. Le rôle de l'expertise dans la construction du jugement est multiple, variable et produit des situations contrastées. Différents facteurs qui président à l’organisation de cette

\footnotetext{
${ }^{80}$ Voir Laurence DUMOULIN, « La médecine légale aux fondements de l'expertise judiciaire, de l’activité de médecin légiste à la profession d'expert », communication au colloque Homo-criminalis : Pratiques et théories médico-légales (XVIe-XXe siècles), Association internationale pour l'histoire du crime, Genève, juin 1997, actes à paraître.

${ }^{81}$ Entretien avec un juge d’instruction, TGI de Grenoble, 3 juin 1998.
} 
diversité peuvent être isolés. Ils sont susceptibles d'expliquer cette réalité plurielle qui donne à voir une plus ou moins grande adéquation entre rapport d'expertise et décision judiciaire.

\subsection{Expertise et décision : des relations à géométrie variable}

La nature et le statut social de la discipline représentent certes une variable capitale pour expliquer le rôle de l'expertise dans l'élaboration du jugement. Immédiatement parlant pour les acteurs et régulièrement évoqué, ce découpage sciences exactes / sciences humaines peut cependant être doublé d'une distinction plus fine relative à la nature des opérations effectuées par l'expert.

Loin d'être monophonique, le discours expert met en scène des opérations intellectuelles multiples qui se positionnent le long d'un continuum dont les pôles extrêmes sont l'observation, la constatation, la description, le relevé, le calcul et la mesure d’un côté ; l’interprétation, l'évaluation et la mise en intelligibilité de l'autre côté. Quelque part entre ces deux polarités se trouve l'opération de diagnostic qui assure le passage, l'articulation entre l'analyse d'un cas concret et le savoir préexistant.

Cette dichotomie "poreuse" a le mérite de permettre de penser une différence de statut des opérations effectuées et des résultats obtenus dans le cadre d’une même spécialité. De ce point de vue, elle semble plus riche et plus heuristique que le découpage traditionnel entre disciplines scientifiques et sciences humaines. Par ailleurs, elle a d'autant plus de sens que l'acte d'expertise est un acte spécifique qui a ses propres exigences. Les façons de faire, comme les objectifs, diffèrent sensiblement en situation d'expertise médicale et dans une consultation traditionnelle par exemple.

Ce découpage extra disciplinaire permet de reconnaître à certains actes qui participent de l’expertise en sciences humaines, une dimension scientifique au sens poppérien du terme ${ }^{82}$. Cette catégorie met sur un pied d’égalité la biologie, la physique, les mathématiques, les techniques dérivées de ces disciplines comme la photographie et plus largement les opérations de calcul, de

\footnotetext{
${ }^{82}$ Voir Karl R. POPPER, Conjectures et réfutations. La croissance du savoir scientifique, Paris, Payot, 1985.
} 
comptage, de dosage, de mesure, de relevé de cotes ou de dessin de plans. Les procédures de prélèvement et les activités de constatation et de description répondent également au critère de falsificabilité de Popper. A défaut d’être vrais, ces actes sont vérifiables et falsifiables. Dans une autre catégorie entrent des disciplines ou techniques dans lesquelles l'interprétation et la subjectivité jouent un rôle considérable. Il s’agit principalement des sciences humaines et des sciences appliquées (psychologie, médecine) et plus largement des activités de nature interprétative. Au sein du corpus étudié, il semble que la pratique des magistrats ait tendance à concéder un rôle important voire déterminant aux actes constitutifs du rapport d'expertise qui entrent dans la première catégorie alors que l'utilisation des autres items serait moins systématique et ferait jouer d'autres facteurs.

A partir du moment où les items qui composent le rapport d'expertise n’apportent pas d'affirmations falsifiables - vues comme des certitudes négatives ou positives - les acteurs judiciaires retrouvent leur marge d’appréciation, leur latitude d’action. Délivrés de la dimension contraignante, ils retrouvent le rapport d'expertise comme ressource, dont l'utilisation dépend alors d'autres facteurs.

Une certaine expérience du tandem, l'existence de liens de confiance entre magistrat et expert, la capacité du technicien à se glisser dans le moule du "bon expert", semblent favoriser un usage massif des conclusions expertales. On rejoint ici l'idée de couples magistrat-expert relativement stables et fonctionnant selon un modèle assez routinier ${ }^{83}$.

A l’opposé, le Tribunal peut fort bien défendre et utiliser un rapport d'expertise dont il considère qu’il est entaché d’erreurs procédurales, qu’il est de mauvaise qualité ou que son auteur manque de professionnalisme. Dans un litige de la construction ${ }^{84}$, l'ingénieur désigné comme expert est brutalement mis en cause par un des avocats qui demande l'annulation du rapport,

\footnotetext{
${ }^{83}$ Danièle BOURCIER et Monique de BONIS ont déjà mis en évidence l'existence de ces "couples" dans Les paradoxes de l'expertise. Savoir ou juger ?, op. cit. Le Plessis-Robinson, Institut Synthélabo pour le progrès de la connaissance, coll. « Les empêcheurs de penser en rond », 1999.
} 
s'appuyant notamment sur le fait que l'expert s'est adjoint de son propre chef deux sapiteurs ${ }^{85}$, ce qui serait constitutif d'un vice de forme. Face à cette mise en cause explicite de l'expertise, le magistrat s’est efforcé de défendre la position de l'expert et ce, bien qu’il ait considéré que les manières de faire du technicien n’aient pas été des plus judicieuses. Il semble - après entretien avec lui - qu’il ait rejeté la demande de nullité du rapport, avant tout pour des considérations d’opportunité et d’équité, construisant ensuite l'argumentation pertinente pour servir cet objectif. Ordonner une nouvelle mesure d’instruction aurait à coup sûr provoqué un prolongement excessif de l'instance ainsi qu'un accroissement démesuré du coût financier assumé par les parties. Aussi a-t-il soutenu un rapport d’expertise dont il reconnaît en privé qu’il pouvait présenter certains défauts de procédure. Ce souci de l'efficacité, du gain de temps peut également expliquer que des rapports d'expertise ouvertement contestés ou critiqués par le magistrat dans le jugement soit néanmoins utilisés comme supports et outils de ce même jugement.

En somme, si la nature des opérations accomplies par l’expert et leur "dureté" - au sens d’aptitude à apporter des certitudes - sont déterminantes pour leur utilisation ultérieure dans la construction du jugement, d'autres variables ont également une certaine influence : le caractère plus ou moins routinier du litige, la relation qui unit le magistrat à l'expert, la capacité du technicien à correspondre au modèle de l'expert idéal, les considérations d’opportunité propres au contexte de l'affaire.

\section{Conclusion : Le droit à l'épreuve des savoirs}

Vue par le droit comme une pure technique, un outil inerte, l'expertise est en réalité une procédure active, interactive et réactive, dont l’émergence dans le champ judiciaire n’est ni anodine ni inoffensive. La Justice entre dans un rapport d'interrelation avec un corps et une logique

\footnotetext{
${ }^{84}$ Dossier B6.

${ }^{85}$ Le sapiteur est un spécialiste que l’expert désigné consulte afin qu’il lui apporte une aide ponctuelle dans la réalisation de sa mission. Ce technicien doit cependant «avoir une spécialité distincte de celle de l'expert », Michel OLIVIER, «Mesures d'instruction confiées à un technicien », op. cit. p. 46. Il s'agissait, en l'occurrence, d’un ingénieur et d'un expert-comptable.
} 
profanes. Il en résulte une transformation de l'économie du système décisionnel, les savoirs agissant comme ressource mais aussi comme contrainte imposée, susceptible de structurer voire de déterminer le jugement, au moins dans un certain nombre de litiges. Les efforts législatifs, réglementaires, doctrinaux pour maintenir le droit dans une position dominante ne parviennent pas à enrayer cette évolution des manières de juger et des fondements de la décision. Le pouvoir du magistrat se heurte au pouvoir de l'expert, avec lequel il lui faut nécessairement composer, tant pour améliorer la qualité du service rendu par la Justice que pour légitimer, aux yeux des justiciables, l’action judiciaire.

"Procédure rationalisante", l'expertise donne les moyens au juge de mener un examen informé des contentieux et apporte des garanties quant au traitement des dossiers. "Procédureraccourci", elle propose des avis et solutions clefs en main, que le magistrat est susceptible de valider tels quels - surtout dans le contexte d'une Justice surchargée et manquant de moyens plutôt que de faire l'effort de construction d’un jugement distinct. "Procédure enfermante", elle produit des résultats susceptibles de contraindre le magistrat et de le placer dans une position non plus de décideur mais de superviseur, c'est-à-dire d'instance de validation de décisions pré-établies au stade de l'expertise.

Dans ce jeu classiquement réglé par les dispositifs normatifs, des principes et moyens d'action concurrents mettent donc en péril la traditionnelle «force du droit » ${ }^{86}$ ainsi que l'autonomie de ses praticiens. Certes, la loi constitue l'irréductible toile de fond de l'action judiciaire de même qu'elle représente toujours une source d’inspiration pour le juge. Il n’en demeure pas moins que le droit semble connaître aujourd'hui une crise profonde. Jusque-là «vecteur d'une entreprise de rationalisation », il en « devient lui-même objet »" ${ }^{87}$ A la rationalité

\footnotetext{
${ }^{86}$ Pierre BOURDIEU, « La force du droit. Eléments pour une sociologie du champ juridique », Actes de la recherche en sciences sociales, $n^{\circ} 64,1986$, p. 3-19.

${ }^{87}$ Jacques CHEVALLIER, « La rationalisation de la production juridique », in Charles-Albert MORAND (dir.), L'Etat propulsif. Contribution à l'étude des instruments d'action de l'Etat, Paris, Publisud, 1991, p. 11.
} 
juridique succède une «rationalité technico-économique » ${ }^{88}$ centrée sur la notion d'efficacité. Le développement de l'expertise participe de ce paradigme managérial ${ }^{89}$ et concourt activement à la fragilisation de la Raison juridique. Sensée rechercher le vrai pour établir le juste, la Justice sollicite des sources extérieures de vérité. En dépit de résistances farouches, ces discours de vérité, convoqués pour dire le vrai, livrent une lecture de la réalité qui tend à conditionner l'application du droit. Ils contribuent ainsi à dessiner une palette renouvelée des pouvoirs et des rôles du magistrat. Fonctionnant comme un système de ressource-contrainte, le recours aux savoirs produit et traduit à la fois une certaine mutation des fondements et modes d'action de l’institution judiciaire.

\footnotetext{
${ }^{88}$ Ibid. p. 12.

${ }^{89}$ Voir notamment Jacques CHEVALLIER et Danièle LOSCHAK, « Rationalité juridique et rationalité managériale dans l'administration française », Revue française d'administration publique, n²4, 1982, p. ainsi que Patrice DURAN, « Piloter l'action publique avec ou sans le droit ? », in Politiques et management public, n4, 1993, p. 2-45 et, du même auteur, Penser l'action publique, Paris, LGDJ, coll. « Droit et société », 1999.
} 


\section{Pour en savoir plus...}

Robert CASTEL, « Savoirs d'expertise et production de normes », in François CHAZEL et Jacques COMMAILLE, Normes juridiques et régulation sociale, Paris, LGDJ, 1991, p. 177-188.

CRESAL, Les raisons de l'action publique. Entre expertise et débat, Paris, L’Harmattan, 1993.

Thomas L. HASKELL (dir.), The Authority of Experts, Bloomington, Indiana University Press, 1984.

Christian PECQUEUR, «Les sciences, auxiliaires du droit ? », Autrement, série Mutations, n¹45, 1994, p. 43-50.

Jean-Yves TREPOS, La sociologie de l'expertise, Paris, PUF, coll. « Que sais-je ? », 1996. 


\section{Résumé}

Depuis plus d'un siècle, la Justice s'est adjoint des partenaires d'un genre spécifique : les experts. Bien que banalisé par le discours juridique, ce recours à des savoirs et savoir-faire extérieurs interroge fortement les fondements de la Justice. Dès lors que des logiques profanes sont introduites au cœur du judiciaire, qu'en est-il du monopole du droit dans la définition du juste ? En dépit des "impératifs" énoncés par les dispositifs formels, l’expertise n’est pas une procédure inerte. Elle participe pleinement de l'évolution des manières de juger et des fondements de la décision. Fonctionnant comme un système de ressource-contrainte, susceptible d'être instrumentalisée par les acteurs judiciaires mais aussi de les contraindre et de peser sur la construction du jugement, l'expertise, en tant que forme d'introduction des savoirs dans l'espace judiciaire, contribue à dessiner une palette renouvelée des pouvoirs et des rôles du magistrat.

Droit - Expertise - Justice - Jugement - Rationalité - Savoirs.

\section{Summary}

Justice and Knowledge : Expert Appraisal in the Formation of the Judgement

For over a century, Justice have been working with a special sort of partner, namely the expert witnesses. Whereas law intends maintaining strict control over them, expert witnesses play a important role in the judicial decision-making process. Expert reports are not only sources of ideas and arguments for the actors in the judicial scene, they are also able to establish certainties. For lawyers and judges, expert appraisal functions as a resource but also as a constraint. Expert evaluation can be decisive in the pronouncement of a judgement. Legal efforts to maintain the dominance of the law are not sufficient to abate the evolution of ways of judging and the grounds of decision. In this interplay usually regulated by law, rival principles of action endanger the traditional « force of law », bringing into question the role and scope of legal settlement.

Expert witnesses - Expert appraisal - Judgement - Knowledge - Law - Rationality. 


\section{L'auteur}

Doctorante au Centre de recherche sur le politique, l'administration, la ville et le territoire (CERAT), Institut d'études politiques, à Grenoble, elle prépare une thèse de Science politique sur l'expertise comme nouvelle raison politique en prenant pour terrain d'études le cas de l'expertise judiciaire. Avec Frédéric Chauvaud, elle a collaboré au rapport Experts et expertises judiciaires en France (1791-1944) réalisé dans le cadre d’un contrat de recherche avec le GIP-Justice et participe par ailleurs à un contrat de recherche sur les pratiques et politiques de gestion de la violence à l’école primaire.

Elle a publié :

- « La mosaïque de l’expertise judiciaire : entre public et privé, monopole et concurrence », Cahiers de la Sécurité intérieure, n³4, 1998, p.233-251 .

- avec Jacques COMMAILLE et Cécile ROBERT (dir.), La juridicisation du politique. Leçons scientifiques, Paris, LGDJ, coll. « Droit et société. Recherches et Travaux », 2000.

- avec Cécile Robert, « », in Jacques COMMAILLE, Laurence DUMOULIN et Cécile ROBERT (dir.), La juridicisation du politique. Leçons scientifiques, Paris, LGDJ, coll. « Droit et société. Recherches et Travaux », 2000, p.

- avec Thierry DELPEUCH, «La Justice : émergence d'une rhétorique de l'usager », in Philippe WARIN (dir.), Quelle modernisation des services publics ? Les usagers au cour des 